\title{
Reliability and Energy Output of Bifacial Modules
}

\author{
Bas B. Van Aken, Mark J. Jansen and Nico J.J. Dekker \\ ECN - Solar Energy, P.O. Box 1, 1755 ZG Petten, the Netherlands
}

\begin{abstract}
Although flash tests under standard test conditions yields lower power due to transmittance of the back sheet, bifacial modules are expected to outperform their monofacial equivalents in terms of yearly energy output in the field. We compare flash tests for bifacial modules with and without a light scattering panel directly behind the modules: $3 \%$ more power output is obtained. We also report on the damp-heat reliability of modules with transparent back sheet. Finally we will present the results of an outdoor study comparing modules with transparent back sheet and modules with state-of-the-art AR coating on the front glass.
\end{abstract}

Index Terms - accelerated aging, degradation, optical properties, photovoltaic systems, silicon, solar power generation.

\section{INTRODUCTION}

The design of ECN's n-Pasha cell, which is manufactured by Yingli Solar, is bifacial. To fully utilise the potential of this bifacial n-type solar cell concept, the rear side of n-type modules has to be transparent to harvest the indirect light. The amount of indirect light falling onto the rear side of the module will depend on the module surroundings, for instance a reflective surface.

The requirements with respect to durability for the transparent back sheet are the same as for standard white or black back sheets including compatibility with the n-type cells and other module materials [1]. We will present reliability testing of modules with bifacial H-pattern (n-Pasha) cells with a transparent back sheet focussing on the prevention of degradation in damp-heat conditions, particularly the influence of EVA and/or soldering flux.

In a laboratory module flash tester, under standard test conditions which include fully light-absorbing surfaces, the use of a transparent back sheet lowers the peak power output by $3 \%$ compared to a white back sheet due to the lack of reflection at the rear of the cells and between the cells. Additionally, advances in AR coating and texturing of glass sheets increases the light collection by the module. Measured cell-to-module changes of the Isc will be related to the choice of glass and back sheet. Additionally, in this paper, the effect on flash test power output for these modules when the back reflecting panel is varied will be presented. The power loss is fully recovered by placing a white sheet directly behind the module. A power gain is observed in case of placing a white back reflector at some distance behind the module.

The outdoor annual energy output and the $\mathrm{kWh} / \mathrm{Wp}$ of bifacial modules are higher than monofacial modules, with claimed values varying between 5 to $30 \%$, depending on climatic conditions and the local albedo [2]. However, actual values supported by data in the literature are relatively sparse; a recent example by Sanyo reports $+11 \%$ [3] and Kreinin mentions 30\% [2]. We will present the outdoor energy output and bifaciality gain for our modules.

\section{EXPERIMENTAL DETAILS}

Single cell laminates were built to test the reliability of transparent back sheet under damp-heat conditions. The laminates were fabricated using three bus bar n-type monocrystalline Si bifacial n-Pasha solar cells from a single processing batch with average efficiency $\eta=19.5 \%$ [4]. Interconnection was made by soldering tabs to the bus bars and cross-connecting the tabs from each side. Four probe measurements were enabled by soldering two bussing connectors to each cross-connector. Laminates were made either with EVA or a thermoplastic encapsulant. Three back sheets were used, one standard white back sheet with a water vapour transmission rate (WVTR) of $\sim 0.7 \mathrm{~g} / \mathrm{m}^{2} /$ day and two transparent back sheets with WVTR of $\sim 2$ and $\sim 0.3 \mathrm{~g} / \mathrm{m} 2 /$ day.

During damp-heat (DH) climate chamber testing the modules are kept at $85 \%$ relative humidity and $85^{\circ} \mathrm{C}$ for 500 hours per cycle. After each cycle, the power output is measured in an AAA solar simulator and changes in performance are related to observed changes in the electroluminescence (EL) images.

72-cell modules were manufactured for power output measurements under STC and energy output determination when placed outdoors. The full-size modules were made using six 12-cell strings made of solar cells of the same batch as above. Cells were binned on efficiency. Variations in $\mathrm{I}_{\mathrm{mpp}}$ and $\mathrm{V}_{\mathrm{mpp}}$ from the module average were very small. The laminates were made using two glass types: standard textured solar glass or solar glass with state-of-the-art anti-reflection coating to maximise the light coupling. Monofacial and bifacial modules were created with white and transparent back sheet, respectively. All other module aspects were kept the same.

Outdoor measurements are performed on the roof of an ECN building, located at 52 $47^{\prime} 6^{\prime \prime} \mathrm{N}, 4^{\circ} 40^{\prime} 25^{\prime}$ ' E using a clamping system. The location is characterised by close proximity to the North Sea and no shadow. Direct irradiation will be measured with a Pyranometer and reference cell in the plane of the rack, as shown in Fig. 1; note the albedo of the wall and floor behind the modules is rather low. The horizontal light intensity, a measure for the amount of indirect light, is measured with a second Pyranometer. 


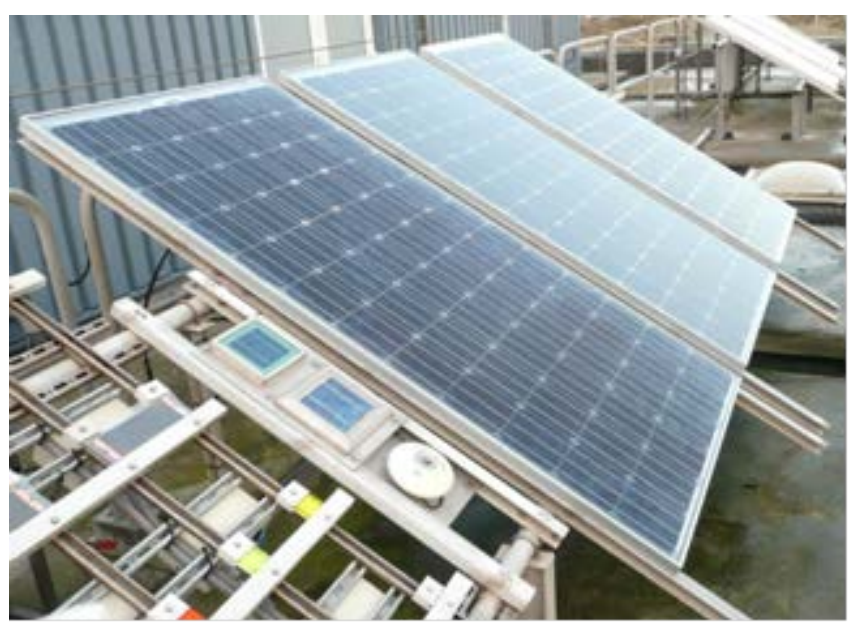

Fig. 1. The three modules on the roof top rig. Reference cells and in-plane Pyranometer are on the foreground. (left-to-right) modules $\mathrm{C}, \mathrm{B}$ and $\mathrm{A}$, see table 1 for details. The modules are tilted at $30^{\circ}$ and their azimuth is $170^{\circ}$, almost due south.

The outdoor measurement system is set up to record an IV-trace per module every 10 minutes and logs the irradiation, ambient temperature and module temperature. During the next 10 minutes the modules are kept at their respective measured Vmpp. The three modules will be evaluated on the amount of energy produced relative to the STC Watt-peak rating due to differences in module build-up. In particular, we will look at the differences when a) the irradiation is at low angles (early/late hours) and b) for bright, but diffuse light situations to determine the increased energy production of the modules with transparent back sheet and the antireflection textured glass relative to the standard lay-up with white back sheet and conventional texture.

\section{RESULTS AND DISCUSSION}

\section{A. Single cell laminates}

Table 1 presents the cell-to-module (CtM) changes in Isc for the single cell laminates. Both solar cells and laminates are measured on the same solar simulator; solar cells are measured on a reflective, conductive chuck, the single cell laminates are isolated from the chuck by blue tissue paper. Clearly laminates with a white back sheet (circles), have a much higher gain in Isc due to reflection and scattering on the white back sheet. For the bifacial laminates the average CtM change for the Isc is $+2.3 \pm 0.3 \%$.

We also see that laminates with thermoplastic encapsulant outperforms the laminates with EVA encapsulant. Table 1 shows that the change in Isc is $+2.0 \pm 0.2 \%$ and $+2.5 \pm 0.3 \%$, respectively for EVA and thermoplastic encapsulant. The difference of $0.5 \%$ in CtM change for the Isc is most likely due to a small difference in optical properties between the two encapsulants.

Table 1. Average cell-to-module change of Isc for single cell laminates for the three types of back sheet (BS) and the two applied encapsulants (encaps).

\begin{tabular}{|c|c|c|}
\hline BS encaps & EVA & TP \\
\hline white & $4.3 \%$ & $6.9 \%$ \\
\hline transparent-1 & $2.0 \%$ & $2.4 \%$ \\
\hline transparent-2 & $2.1 \%$ & $2.6 \%$ \\
\hline
\end{tabular}

Single cell laminates have been exposed in the climate chamber to DH-conditions. Fig. 2 shows the relative fill factor for bifacial laminates as a function of the DH exposure time. For each laminate, the measured fill factor is normalised to the fill factor after lamination. Laminates with thermoplastic encapsulant show only a limited degradation of the fill factor compared to as-laminated. Furthermore, the relative fill factor seems to stabilise at $\sim 98 \%$. In contrast, laminates with EVA show a much larger decrease in fill factor and the fill factor continuous to decrease with further $\mathrm{DH}$ exposure. The $\mathrm{DH}$ results are fully in agreement with control samples fabricated with white back sheet. This indicates that these types of back sheet have no detrimental effect on the DH-resistance of laminates.

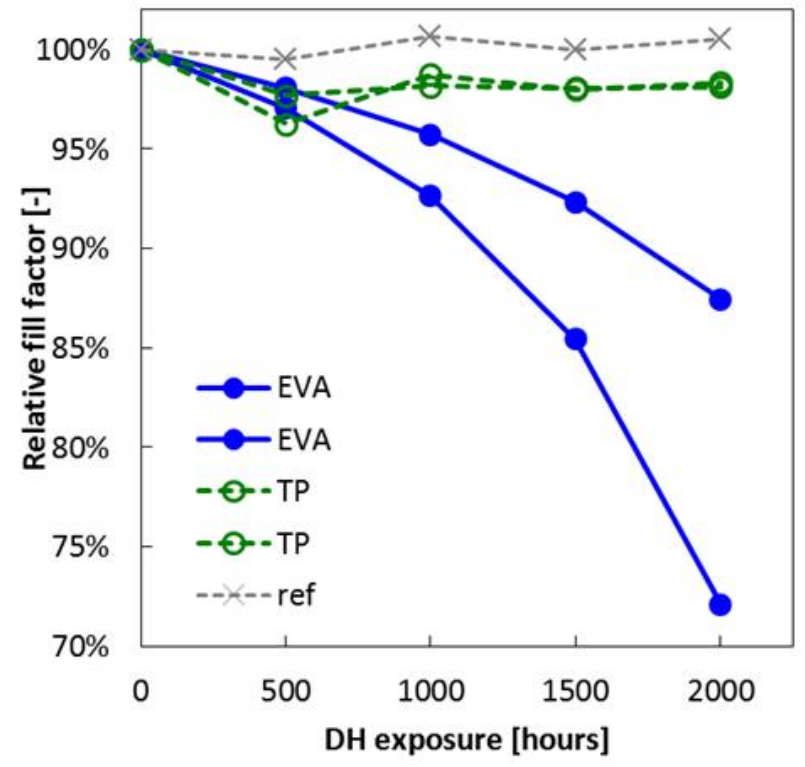

Fig. 2. Normalised fill factor as a function of DH exposure for laminates with EVA (blue, solid) or thermoplastic encapsulant (green, open). The reference laminate with EVA (grey, crosses) has not been put in the climate chamber.

EL images are presented in Fig. 3. The top (bottom) row shows the series for a laminate with EVA (thermoplastic) 
encapsulant after 0, 1000 and 2000 hours of DH exposure. Clearly, loss of EL signal is observed for the EVA sample. The loss originates from the edges of the sample and propagates faster along the bus bars.
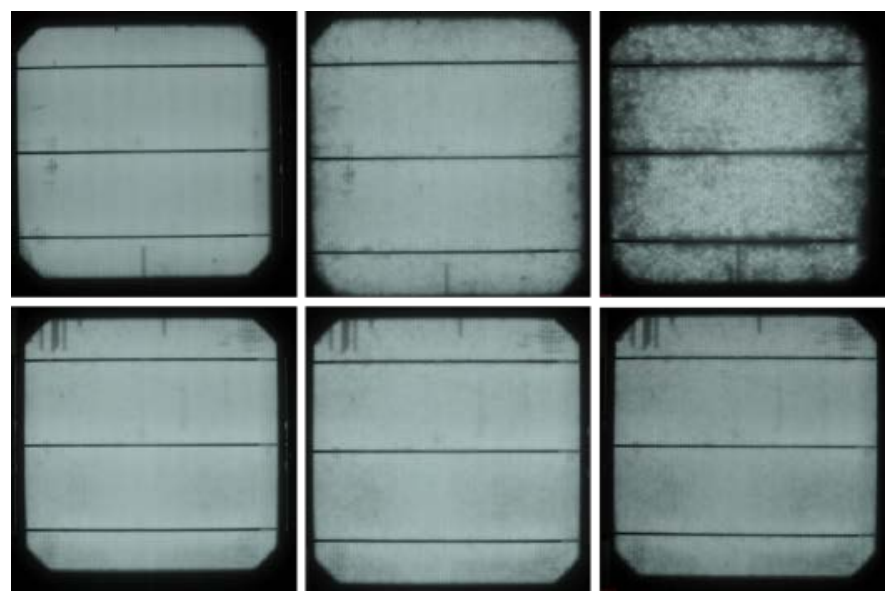

Fig. 3. EL images of two bifacial laminates with EVA (top) and thermoplastic (bottom) encapsulant. Left, middle and top column are taken after 0, 1000 and 2000 hours of DH exposure.

These results do not depend on the type of back sheet. Both types of transparent back sheet and the standard white back sheet show degradation of fill factor when used in combination with EVA and very limited degradation for the thermoplastic encapsulant laminates. The degradation is ascribed to corrosion of the front metallisation that occurs in the presence of acetic acid (from the EVA encapsulant) or solder flux remains. Using thermoplastic encapsulants or otherwise avoiding the presence of acid prevents corrosion of the $\mathrm{Ag} / \mathrm{Al}$ metallisation fingers.

\section{B. 72-Cell modules}

The power of a module can be estimated from the average cell efficiency and gains and losses due to the interconnection and module lay-up. Combining the effects of the $\mathrm{Cu}$ tabs' resistance and the optical effects of glass and EVA, we estimate 3\% power loss for a conventional soldered module with white back sheet and solar glass, i.e. it will have $97 \%$ power compared to the total power of the incorporated solar cells. Table 2 shows the cell-to-module power ratio for the 72-cell modules.
Table. 2. Observed cell-to-module power ratio for modules with conventional/AR textured glass (columns) and white/transparent back sheet (rows). Modules are identified by the capital letter. The value in brackets is calculated, see text.

\begin{tabular}{|c|c|c|}
\hline back sheet & $\begin{array}{c}\text { conventional } \\
\text { texture }\end{array}$ & AR texture \\
\hline white & $(97 \%)$ & A: $99.1 \%$ \\
\hline transparent & B: $93.9 \%$ & C: $96.0 \%$ \\
\hline
\end{tabular}

Using state-of-the-art AR textured solar glass, module A in Table 2, yields a relative improvement of $2 \%$ in Isc and thus peak power. In contrast, applying a transparent back sheet will lead to a decrease in Isc and power when measured under standard test conditions that means no reflection from the background. We estimate that the transparent backsheet leads to about 3\% power drop due to optical losses. The observed values, shown in Table 2, are in excellent agreement, less than $1 \mathrm{~W}$ difference, with these estimates. For instance module B has $2.1 \%$ lower $\mathrm{CtM}$ power ratio compared to module $\mathrm{C}$, due to the lack of AR textured glass.

\section{Bifacial measurements}

To emulate the effect of light scattering and reflecting from a background, we have measured two bifacial modules with a white back sheet at various distances behind the module. The back sheet had the same dimensions as the module, thus only light that is transmitted through the module can be scattered or reflected back to the module. Results are shown in Fig. 4.

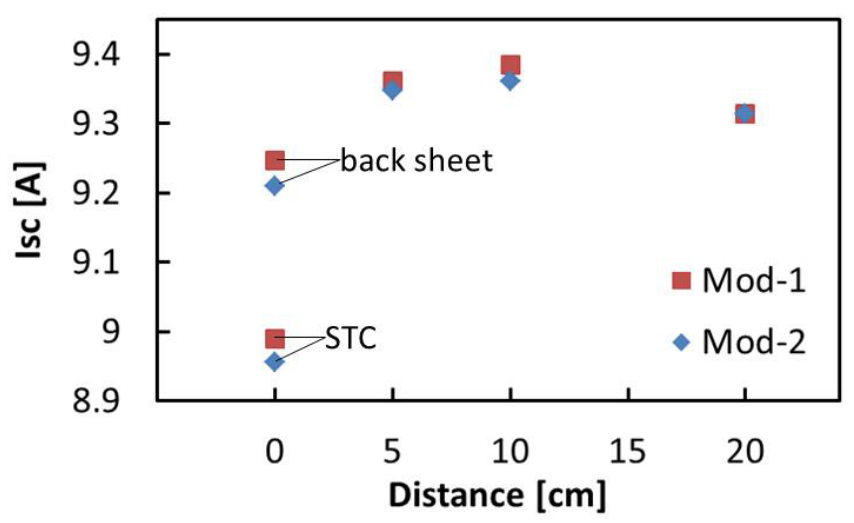

Fig. 4. Isc of bifacial modules without (STC) and with back sheet placed at various distance behind the module.

First, we observe a $3 \%$ increase in $I_{s c}$ when the back sheet is placed at $0 \mathrm{~cm}$ behind the bifacial module. Secondly, placing the back sheet further behind the module initially increases the $\mathrm{I}_{\mathrm{sc}}$ further, but at larger distances the $\mathrm{I}_{\mathrm{sc}}$ decreases slightly. The $3 \%$ increase in $\mathrm{I}_{\mathrm{sc}}$ is in good agreement with the $3 \%$ difference in CtM between bifacial and monofacial modules. The further increase is due to the scattering angles and 
associated distances that the reflected light has to travel. At some distance, light scattered under an angle will hit the open rear side of the n-type cells, whereas at very small distances, this light would have missed the cells. At $20 \mathrm{~cm}$ and beyond, more and more of the transmitted light will not reach the back sheet.

\section{Outdoor measurements}

The outdoor data presented here are accumulated over three months in the winter/early spring of 2013. This period in the Netherlands is typically either overcast, grey with little, mostly indirect light or clear skies with mostly direct light, albeit with rather low irradiation due to the sun's position low in the sky. The plot of the annual distribution of irradiation/energy in Petten, NL, as function of irradiation interval (in $50 \mathrm{~W} / \mathrm{m}^{2}$ bins) exhibits a gradual incline with a broad, shallow peak at 900-950 W/m ${ }^{2}$ [5]. The corresponding plot of energy production for only three months in winter, see Fig. 5, looks rather similar. However, there is a larger contribution of the $100-250 \mathrm{~W} / \mathrm{m}^{2}$ intervals, indicating the abundance of low, light conditions that probably have a relatively large indirect fraction.

In Fig. 5 we have normalised the energy production to each module's peak power as measured under standard test conditions. The bifacial module has a peak power under STC $\mathrm{Wp}=314 \mathrm{~W}$, and the monofacial module has $\mathrm{Wp}=334 \mathrm{~W}$. The figure shows the normalised energy production for a bifacial module and a monofacial module as a function of the in-plane irradiation with $50 \mathrm{~W} / \mathrm{m}^{2}$ bins. Whereas the monofacial module has a total normalised energy production in these three months of $248 \mathrm{Wh} / \mathrm{Wp}$, the bifacial one has 258 $\mathrm{Wh} / \mathrm{Wp}$; an increase of $4 \%$, for a location with far from optimal conditions for back reflection of light.

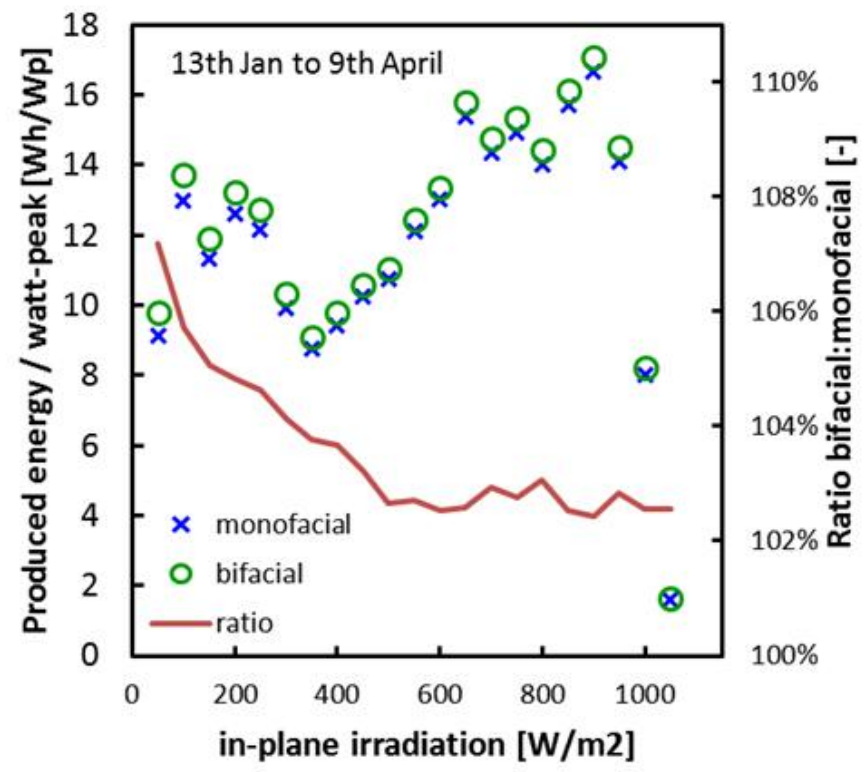

Fig. 5. Energy production for two modules on the roof of the ECN building as a function of the measured in-plane irradiation for a monofacial (blue crosses) and bifacial module (green circles). The produced energy is scaled to the module power under standard test conditions. The ratio of the bifacial/monofacial values is plotted on the right axis (red line).

Fig. 5 shows that the normalised energy production of the bifacial module is higher than that of the monofacial module. The ratio of the normalised energy production of the bifacial and monofacial module is plotted as the red line in Fig. 5. For irradiation levels $>500 \mathrm{~W} / \mathrm{m}^{2}$ the bifacial module has a $3 \%$ larger normalised energy production. At lower irradiation levels the ratio is higher, up to $7 \%$ for the lowest irradiation bins. As these irradiation levels correspond to situations with a larger proportion of indirect light, we assume that this confirms the expectation that bifacial modules outperform their monofacial counterparts under light conditions with a large fraction of indirect light.

Note that in winter time, indirect light only occurs at low light conditions. In contrast, in summer both the in-plane irradiation and the indirect fraction can be high on bright, but overcast days. We expect that on those days, the bifacial:monofacial ratio will be higher than the observed $3 \%$ increase at irradiation $>500 \mathrm{~W} / \mathrm{m}^{2}$ in winter. Consequently, we assume that when data collected over the summer months will be included, the observed increase at lower irradiation bins will shift to higher irradiation. Thus the total normalised energy production of the bifacial module will be more than $4 \%$ higher than the monofacial module even though this roof top location has limited reflection from the rear. Further experiments are planned to gain detailed insight in the conditions that favour bifaciality and are appropriate for commercial and private PV systems. 


\section{CONCLUSIONS}

The Isc CtM for single cells laminates is slightly higher for thermoplastic encapsulants compared to EVA. This effect seems more pronounced for monofacial modules, but has to be confirmed in test with larger number of laminates. DH results up to 2000 hours indicate that laminates with EVA suffer from continuous fill factor degradation, whereas laminates with thermoplastic encapsulants show only a $2 \%$ decrease in fill factor after 2000 hours.

The power output of 72-cell modules has been estimated as a function of back sheet and glass type. These values were accurate within $1 \mathrm{~W}$. A white back sheet as background during flash testing of bifacial modules recovers the power loss due to the transparent back sheet in the laminate.

Outdoor measurements, taken during three months in winter/early spring, showed that bifacial modules, compared to corresponding monofacial modules, have a 3\% higher normalised energy production; at lower irradiation the increase is even higher. We expect that in the summer months, those higher increases in normalised energy production of 5-7\% will also be observed at higher irradiation.

\section{REFERENCES}

[1] I.J. Bennett and B.B. Van Aken, "Testing compatibility of ntype cells and module materials", in 2nd n-PV workshop, Amsterdam, the Netherlands, 2012.

[2] L. Kreinin, N. Bordin, A.. Karensty, A. Dori and N. Eisenberg, "Experimental analysis of the increase in energy generation of bifacial over monofacial PV Modules”, 26 $6^{\text {th }}$ EUPVSEC 4CO.7.1, 2011.

[3] T. Mishima, M. Taguchi, H. Sakata and E. Maruyama, "Development status of high-efficiency HIT solar cells " Solar Energy Materials and Solar Cells, vol. 95, pp. 18-21, 2011.

[4] I.G. Romijn et al. "Industrial implementation of efficiency improvements of n-type solar cells and modules", $27^{\text {th }}$ EUPVSEC, pp. 533-537, 2012.

[5] N.J.J. Dekker, M.J. Jansen, I.J. Bennett and W. Eerenstein, "Characterisation of full size MWT back contact and H-pattern modules by outdoor IV-tracing measurements and explanation of the difference in energy yield", $26^{\text {th }}$ EUPVSEC 4BV.2.12, 2012. 